\title{
Late Nontraumatic Anterior Dislocation of Intraocular Lens with Complete Capsular Phimosis in Retinitis Pigmentosa
}

\author{
In Sim Ng ${ }^{a}$ Wa Cheong Leong ${ }^{a} \quad$ Li Sun $^{\mathrm{a}, \mathrm{b}}$ Chan Leong ${ }^{\mathrm{a}}$ \\ aDepartment of Ophthalmology, Centro Hospitalar Conde de São Januário, Macao, China; \\ bepartment of Ophthalmology, Huashan Hospital, Fudan University, Shanghai, China
}

\section{Keywords}

Intraocular lens dislocation · Anterior chamber $\cdot$ Retinitis pigmentosa $\cdot$ Capsular contraction syndrome

\begin{abstract}
Intraocular lens (IOL) dislocation is an unusual but serious complication after cataract surgery. We are here to report a rare case which is nontraumatic IOL dislocated and prolapsed into the anterior chamber in a retinitis pigmentosa (RP) patient. Zonular weakness and capsular contraction are considered to be the main contributing factors of IOL dislocation in RP patients. Patients at risk should be recognized and managed accordingly intraoperatively or postoperatively.
\end{abstract}

\section{Introduction}

Intraocular lens (IOL) dislocation is an unusual but serious complication after cataract surgery. Late IOL dislocation is defined as occurring 3 months or later after cataract surgery [1]. While trauma is a common cause of late IOL dislocation [2], and the dislocated lens usually drops posteriorly to the vitreous cavity [3], we are here to report a rare case which is nontraumatic IOL dislocated and prolapsed into the anterior chamber in a retinitis pigmentosa (RP) patient.

\section{Karger ${ }^{\prime \prime}$}




\section{Case Reports in Ophthalmology}

Fig. 1. Slit-lamp examination of the left eye showed ciliary injection, hazy cornea with Descemet's folds, Tyndall (+/-), a dislocated IOL with complete occlusion of the anterior capsular opening was noted in the anterior chamber.

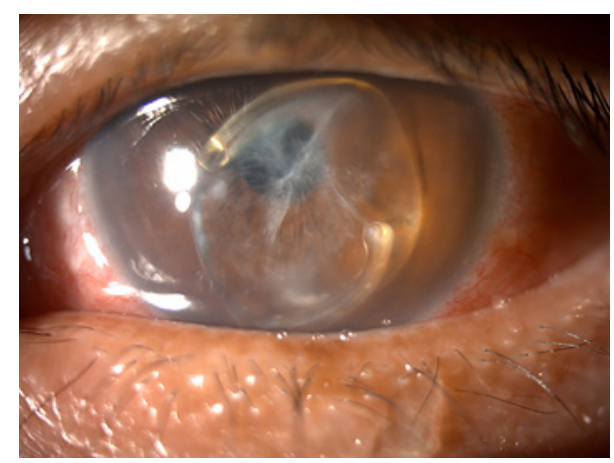

\section{Case Presentation}

A 64-year-old Chinese female presented to our emergency room complaining of pain and redness of her left eye for 1 week, with left-sided headache for 1 day. She denied any history of trauma, but the symptoms began since she had cough following a common cold approximately 1 week ago. She has history of morphea (localized scleroderma) with treatment of hydroxychloroquine $200 \mathrm{mg}$ daily and denied history of other systemic disease. Her ocular history was significant for RP and implantation of posterior chamber IOL OU approximately 5 years ago, inferior subluxation of lens OS was noted during operation. She had regular postoperative follow-up, her visual acuity of left eye was stable at hand motion since operation. On examination of this consultation, her visual acuity was 7/10 OD and hand motion OS. Her intraocular pressure measurements were $14 \mathrm{~mm} \mathrm{Hg}$ OD and $16 \mathrm{~mm} \mathrm{Hg}$ OS with noncontact tonometer. Pupils were equal, round, and reactive to light. Slit-lamp examination of the right eye was unremarkable with a posterior chamber IOL. The left eye showed ciliary injection, hazy cornea with Descemet's folds, Tyndall (+/-), a dislocated IOL wrapped by fibrotic capsular bag was noted in the anterior chamber (shown in Fig. 1), fundus could not be seen. B-scan showed posterior vitreous detachment without evidence of retinal detachment OS. After discussion with the patient, considering the poor vision preoperatively, surgical removal of the dislocated IOL OS without IOL re-fixation or exchange was planned. Therefore, the IOL was removed through a scleral-limbal incision uneventfully.

On postoperative day 1 , the visual acuity was hand motion OS with normal intraocular pressure (13 $\mathrm{mm} \mathrm{Hg}$ by noncontact tonometer). Corneal edema mildly improved, anterior chamber was deep, Tyndall (+), and pupil was round, aphakic, fundus exam showed RP changes, retina flat, cup disc ratio 0.3 .

Two months after operation, the patient had no complaint, her refraction was +16.0 DS/-5.5 DC $\times 40^{\circ}$, best-corrected visual acuity was still hand motion OS, with normal intraocular pressure (17 mm Hg by noncontact tonometer). Cornea was transparent, sutures remained in place, Tyndall $(-)$, the otherwise examination remained same as before.

\section{Discussion}

Late IOL dislocation is defined as occurring 3 months or later after cataract surgery. It is an unusual but serious complication after cataract surgery. Previous studies found an average of 6-9 years after in most cases of uneventful surgery [1]. Our case occurred approximately 5 years after surgery, and the underlying lens subluxation may accelerate the development. While most of the IOL dislocation are related to trauma [2] or other external force such as eye rubbing or poking $[4,5]$, there are some cases occur spontaneously.

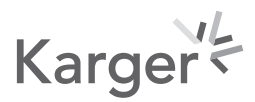


Spontaneous IOL dislocation often occurs in eyes with certain risk factors such as zonular weakness and capsular contraction [6].

Capsular contraction syndrome is a well-known complication after continuous curvilinear capsulorrhexis and presented with shrinkage and whitening of the anterior capsule opening [7] due to anterior capsule fibrosis and phimosis. RP is first reported related to capsular contraction syndrome by Hayashi et al. [8]. They found that anterior capsule contraction in the RP eyes was more extensive than in the control eyes [8]. Progressive constriction of anterior capsule opening result in complications includes pseudophacodonesis, IOL tilt, and decentration. They are thought to be related to the imbalance between the centrifugal zonular force and centripetal force induced by capsular fibrosis of the capsulorrhexis margin [9]. In some cases of RP, even IOL dislocation was described due to zonular laxity, weakness, or dehiscence. Zonular weakness and dehiscence were reported in RP patients $[10,11]$. Lee et al. suggested the accumulation of damage to the zonules from degenerative aging process and minor trauma (e.g., intraoperative traction of zonules) may cause gradual progression of zonular weakness. It may allow progression of the anterior capsular contracture, which may result in further zonular weakness [9]. The abovementioned process eventually becomes a vicious cycle result in IOL dislocation, and it is inferred as the most probable mechanism in our case. Our case presented with a severe form of capsular contraction which caused complete occlusion of the anterior capsular opening, in addition to her underlying superior zonular dehiscence, she was at high risk of IOL dislocation based on the abovementioned factors.

As for the situation that the IOL prolapsed anteriorly into the anterior chamber instead of posterior part, a case report of a nontraumatic anterior dislocation of posterior phakic IOL suggested that nocturnal mydriasis combined with Valsalva maneuver, such as coughing, may have contributed to the IOL being displaced anteriorly [12]. Our patient mentioned the symptoms of eye redness and pain started after cough. The increased IOP during coughing under mydriatic status might have been the leading factor of IOL anterior dislocation.

$\mathrm{RP}$ is a risk factor of nontraumatic IOL dislocation related to zonular weakness and capsular contraction $[10,13]$, other risk factors reported in literatures including pseudoexfoliation syndrome, high myopia, uveitis, diabetes mellitus, and certain connective tissue disorders such as Marfan's syndrome and advanced age $[1,14,15]$. The recognition of these risk factors help physicians to be alert to possible complication so that they can provide a modified approach in cases at risk for prevention, such as a careful preoperative evaluation with well-dilated pupil to find out any zonulopathy, good quality of anterior capsulorrhexis (well centered and as circular as possible with a minimum diameter of $5.0 \mathrm{~mm}$ ) [16], avoidance of nucleus rotation during cataract surgery to minimize stress on zonules, capsular tension ring insertion to support the capsular bag and decrease postoperative capsule shrinkage [14], IOL selection preferable to hydrophobic lenses [17]. After operation, ophthalmologists still need to be aware of the possibility of delayed IOL dislocation which may occur several years after the operation [10]. Anterior capsule radial relaxing incisions with Nd:YAG laser early after cataract surgery are recommended in high-risk patients for prevention of anterior capsular contraction $[18,19]$. RP patients should be informed about the possibility of delayed postoperative complications [10] and should be educated to protect eye by avoiding trauma, eye rubbing, and any situation that can cause increased IOP such as severe coughing spells, vomiting, and Valsalva maneuver.

\section{Statement of Ethics}

Written informed consent was obtained from the patient for publication of this case report and any accompanying images.

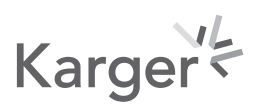




\section{Conflict of Interest Statement}

The authors declare that there are no conflicts of interest to disclose.

\section{Funding Sources}

This research received no specific grant from any funding agency in the public, commercial, or not-for-profit sectors.

\section{Author Contributions}

I.S.N.: writing and reviewing. W.C.L.: writing and reviewing. L.S.: writing and reviewing. C.L.: reviewing.

\section{References}

1 Kristianslund O, Råen M, Østern AE, Drolsum L. Late in-the-bag intraocular lens dislocation: a randomized clinical trial comparing lens repositioning and lens exchange. Ophthalmology. 2017 Feb;124(2):151-9.

2 Hazzazi MA, Rashaed SA. Outcomes and determinants of posterior dislocated intraocular lens management at a tertiary eye hospital in central Saudi Arabia. Middle East Afr J Ophthalmol. 2019 Oct-Dec;26(4):223-8.

3 Hayashi K, Ogawa S, Manabe SI, Hirata A, Yoshimura K. Response to: a classification system of intraocular lens dislocation sites under operating microscopy and surgical techniques and outcomes of exchange surgery. Graefes Arch Clin Exp Ophthalmol. 2016 Mar;254(3):2489-91.

4 Poh EP, Fariza NN, Mariam I. Bilateral anterior chamber intraocular lenses dislocation in a patient with habitual eye rubbing. Med J Malaysia. 2005 Aug;60(3):370-2.

5 Al-Owaid AA, Alarfaj MA, Alarfaj FA, Awad A. Intraocular lens dislocation into the anterior chamber because of repeated eye-poking in a patient with leber's congenital amaurosis. Case Rep Ophthalmol. 2020 Jan;11: 48-53.

6 Gross JG, Kokame GT, Weinberg DV. In-the-bag intraocular lens dislocation. Am J Ophthalmol. 2004 Apr; 137(4):630-5.

7 Al-Kharashi SA, Al-Obailan M. Capsular phimosis with complete occlusion of the anterior capsular opening after intact continuous curvilinear capsulorrhexis. Saudi J Ophthalmol. 2009 Jul;23(2):175-8.

8 Hayashi K, Hayashi H, Matsuo K, Nakao F, Hayashi F. Anterior capsule contraction and intraocular lens dislocation after implant surgery in eyes with retinitis pigmentosa. Ophthalmology. 1998 Jul;105(7):1239-43.

9 Park TK, Chung SK, Baek NH. Changes in the area of the anterior capsule opening after intraocular lens implantation. J Cataract Refract Surg. 2002 Sep;28:1613-7.

10 Lee HJ, Min SH, Kim TY. Bilateral spontaneous dislocation of intraocular lenses within the capsular bag in a retinitis pigmentosa patient. Korean J Ophthalmol. 2004 Jun;18:52-7.

11 Namiki M, Tagami Y, Morino I, Kano M, Sugiura T. [Findings from slit lamp and histological examination of the anterior capsule in patients with severe anterior capsular shrinkage and opacities after implantation of intraocular lenses]. Nippon Ganka Gakkai Zasshi. 1993 Jun;97(6):716-20.

12 Connor M, Downer D, Park HJ, Tuli S. Non-traumatic dislocation of a first generation phakic intraocular lens. Invest Ophthalmol Vis Sci. 2007 May;48(13):5429.

13 Najjar DM, Igbre AO, Tsai FF. Late capsular bag contraction and intraocular lens subluxation in retinitis pigmentosa: a case report. J Med Case Rep. 2011 Feb;5:65.

14 Ascaso FJ, Huerva V, Grzybowski A. Epidemiology, etiology, and prevention of late IOL-capsular bag complex dislocation: review of the literature. J Ophthalmol. 2015 Dec;2015:805706.

15 Choudhary A, Sahni J, Kaye SB. Late spontaneous anterior dislocation of an intraocular lens (IOL) with the capsular bag. Eye. 2005 Jan;19(1):101-2.

16 Davison JA. Capsule contraction syndrome. J Cataract Refract Surg. 1993 Sep;19(5):582-9.

17 Tsinopoulos IT, Tsaousis KT, Kymionis GD, Symeonidis C, Grentzelos MA, Diakonis VF, et al. Comparison of anterior capsule contraction between hydrophobic and hydrophilic intraocular lens models. Graefes Arch Clin Exp Ophthalmol. 2010 Aug;248(8):1155-8.

18 Nikpoor N, Stone DU. Rapid capsular phimosis in retinitis pigmentosa. Ophthalmic Surg Lasers Imaging. 2010 Jul;41. Online.

19 Hayashi K, Yoshida M, Hirata A, Hayashi H. Anterior capsule relaxing incisions with neodymium: YAG laser for patients at high-risk for anterior capsule contraction. J Cataract Refract Surg. 2011 Jan;37:97-103. 